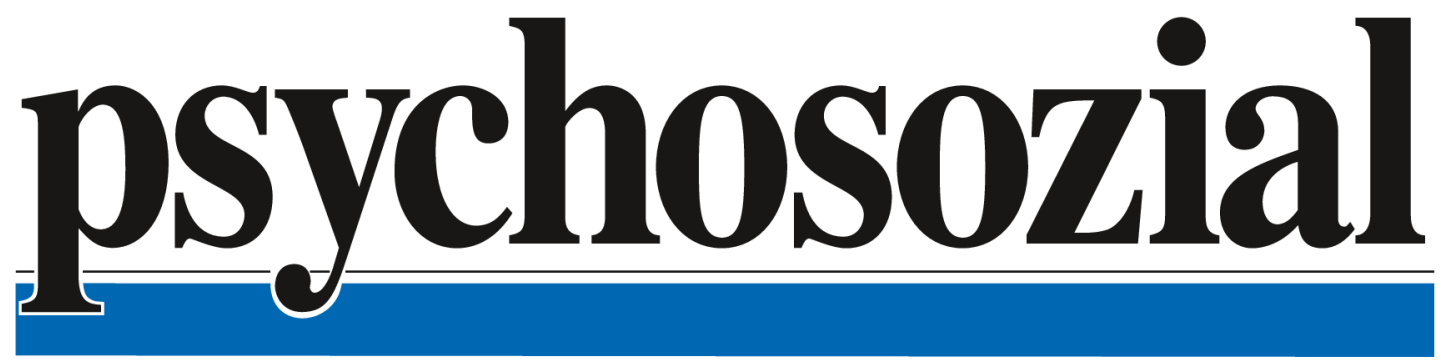

Herausgegeben von Michael B. Buchholz, Pradeep Chakkarath, Oliver Decker, Jörg Frommer, Benigna Gerisch, Rolf Haubl, Marie-Luise Hermann, Vera King, Carlos Kölbl, Joachim Küchenhoff, Jan Lohl, Katja Sabisch, Jürgen Straub, Hans-Jürgen Wirth und David Zimmermann

ISSN 0171-3434・43. Jahrgang • Nr. 162・2020・Heft IV
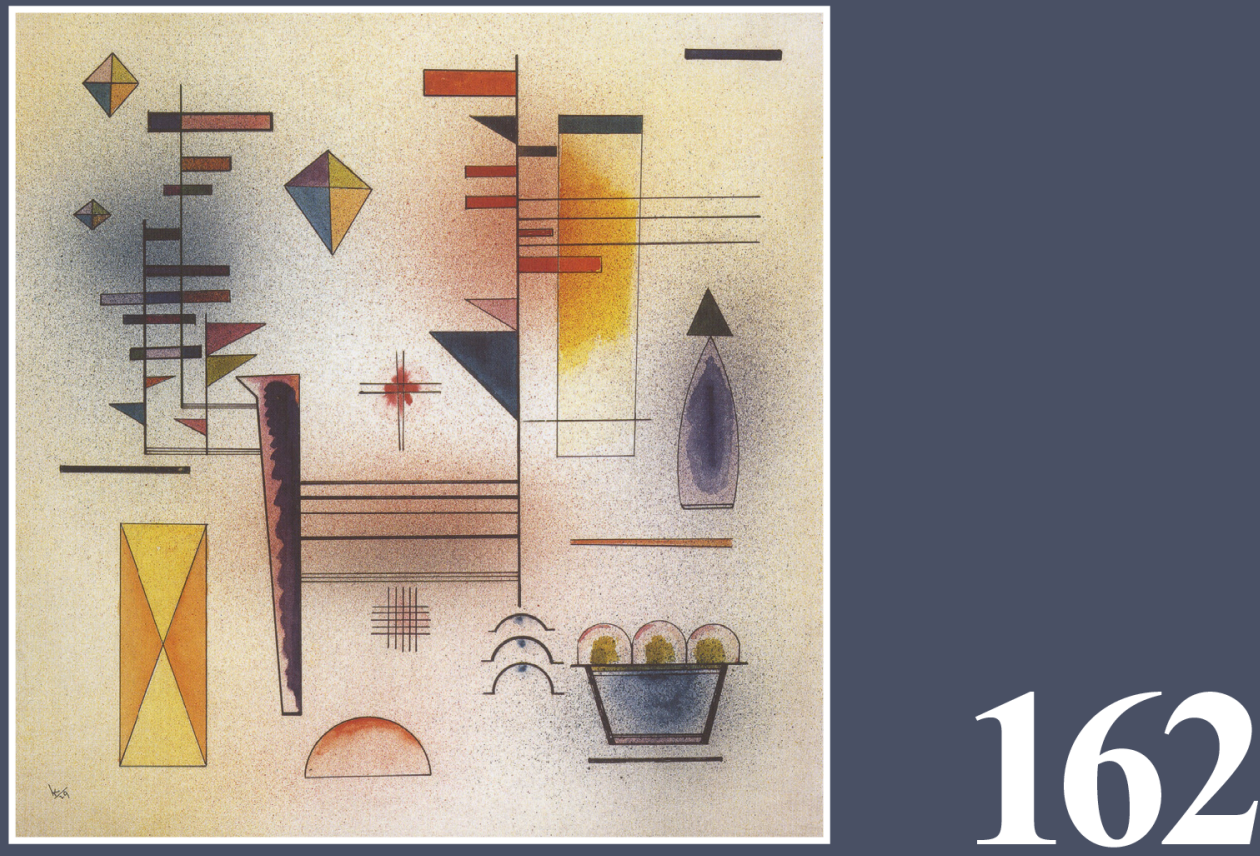

Schwerpunktthema:

\title{
Kritische Lebenskunst
}

Herausgegeben von Günter Gödde, Jörg Zirfas und Hans-Jürgen Wirth 


\section{Inhalt}

Schwerpunktthema:

Kritische Lebenskunst

Möglichkeiten und Grenzen

eines gelingenden Lebens

Alltägliche, gesellschaftliche

und therapeutische Kontexte

Editorial

GÜNTER GÖDDE, JÖRg ZIRFAS

\& HANS-JÜRGEN WIRTH

Lebenskunst in der Therapie

Ein Fallbeispiel

GÜNTER GöDdE \& Jörg Zirfas

Rechtfertigung, Abschaffung, Bejahung

Wege zum Umgang mit Leiden

Helmut Heit

Ungerechtigkeit und Missachtung

Über Erwartungen und Erfahrungen

JöRG ZIRFAS

Echt hässlich!

Über das Nicht-Schöne

in Kunst, Kultur und Psyche

Karin DANNecker

Alltagskunst

Zu einer Psychästhetik des Alltags

Werner Pohlmann

Offensiv leben

Über das Scheitern als gesuchte Chance

Robert ZimMer

Lebenskunst und Narzissmus

Schwierigkeiten und Chancen

des selbstreflexiven Selbst,

das Leben zu meistern

HANS-JÜRGEN WIRTH
Liebe und Sehnsucht

Bedrohungen und Möglichkeiten der Liebe in modernen Zeiten

Silvia SCHNeIDER

5

»Die Zeit heilt alle Wunden «

90

$\mathrm{Zu}$ einem Spruch der (kritischen) Lebenskunst

Werner Stegmaier

Erinnern und Vergessen

in der therapeutischen Lebenskunst

11 GüNTER GöDDE

Psychotherapie und kritische Lebenskunst aus Sicht der Therapieforschung

23 Heinrich Deserno

\section{Freier Beitrag}

33 Überlegungen zu Musik unter Verweis auf ausgewählte Modelle psychischer Gesundheit

ERIC Pfeifer

\section{Rezensionen}

(Selbst-)Kritik und Krisenhilfe

Günter Gödde \& Jörg Zirfas (Hrsg.). (2018).

53 Kritische Lebenskunst. Analysen -

Orientierungen - Strategien

Dagmar Kiesel

62 Anmut, Würde, Leidenschaft -

Lebenskunst revisited

Günter Gödde \& Jörg Zirfas (Hrsg.). (2018).

Kritische Lebenskunst. Analysen -

71 Orientierungen-Strategien

Hans-Gerd von Seggern

Dank an die Gutachterinnen

und Gutachter

Impressum 


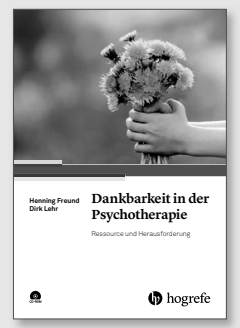

Henning Freund/

Dirk Lehr

Dankbarkeit in der

Psychotherapie

Ressource und

Herausforderung
2020, 217 Seiten, inkl. CD-ROM,

$€ 29,95 /$ CHF 39.90

ISBN 978-3-8017-2893-9

Auch als eBook erhältlich

Das Thema Dankbarkeit erhält in der Psychologie zunehmend Aufmerksamkeit. Viele Studien belegen die hohe Relevanz von Dankbarkeit für die psychische Gesundheit. Das Buch informiert umfassend über den aktuellen Wissensstand rund um Dankbarkeit und stellt zahlreiche Übungen sowie Arbeitsmaterialien zur Förderung von Dankbarkeit vor.

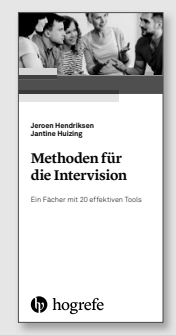

Jeroen Hendriksen/

Jantine Huizing

Methoden für die Intervision

Ein Fächer mit

20 effektiven Tools

2020, 72 Seiten, Kleinformat,

$€ 16,95 /$ CHF 21.90

ISBN 978-3-8017-3033-8

Für die Arbeit in Intervisionsgruppen stellt dieser Fächer zwanzig effektive Arbeitsmethoden vor. Unterschieden werden dabei lösungsorientierte, kreative, aktivierende und reflexive Arbeitsformen. Die vorgestellten Tools geben neue Impulse für die Intervision.

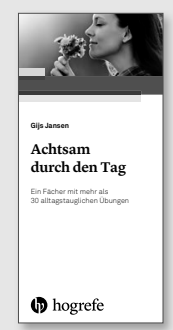

Gijs Jansen

Achtsam durch den Tag

Ein Fächer mit mehr als

30 alltagstauglichen

Übungen
2020, 54 Seiten, Kleinformat,

$€ 16,95 /$ CHF 21.80

ISBN 978-3-8017-3034-5

Dieser Fächer ist ein praktischer Begleiter für alle, die Achtsamkeit praktizieren und sich selbst und ihre Umgebung neu erkunden wollen. Er enthält mehr als 30 Übungen für den Alltag. Achtsamkeit ist keine Frage der Örtlichkeit, Sie können immer und überall achtsam sein. Mit den Übungen lernen Sie vor allem, Ihre Wahrnehmung zu schärfen und offen zu sein für das, was sich im Hier und Jetzt ereignet.

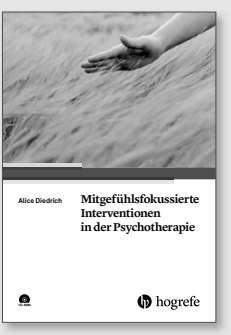

\section{Alice Diedrich \\ Mitgefühls- \\ fokussierte \\ Interventionen in der Psychotherapie}

2016, VIII/152 Seiten, inkl. CD-ROM, $€ 26,95 / \mathrm{CHF} 35.90$

ISBN 978-3-8017-2671-3

Auch als eBook erhältlich

Das Buch stellt Interventionen zur Steigerung von Mitgefühl in der psychotherapeutischen Praxis vor. Übungsanleitungen, Fallbeispiele und Patient-Therapeut-Dialoge illustrieren das Vorgehen.

www.hogrefe.com 
Schwerpunktthema:

\section{Kritische Lebenskunst}

Herausgegeben von Günter Gödde, Jörg Zirfas und Hans-Jürgen Wirth 


\section{Kritische Lebenskunst}

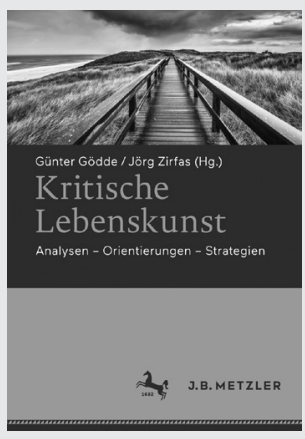

G. Gödde, J. Zirfas (Hrsg.)

\section{Kritische Lebenskunst}

Analysen - Orientierungen - Strategien 2018, XIV, 490 S. 10 Abb., 7 Abb. in Farbe. Geb. $€(D) 49,99$ | $€(A) 51,39 \mid{ }^{*} \mathrm{CHF} 55.50$

ISBN 978-3-476-04643-7

$\rightarrow$ Geschrieben von Expert/innen aus Theorie und Praxis

$\rightarrow$ Sozial und kulturell fokussierte Lebenskunst

$\rightarrow$ Interdisziplinäre Behandlung des Themas

Die Philosophie der Lebenskunst gehört seit Ende des 20. Jahrhunderts zu den aktuellen Denkrichtungen, die nicht nur in Lehre und Forschung vertreten, sondern auch von einem breiteren Publikum wahrgenommen werden. In ihr geht es um ein gelungenes, schönes und glückliches Leben.

$€(D)$ : gebundener Ladenpreis in Deutschland, $€(A)$ : in Österreich. * unverbindliche Preisempfehlung. Alle Preise inkl. MwSt.

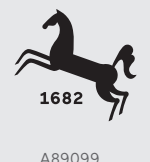

\section{鸪 Psychosozial-Verlag}

Günter Gödde \& Jörg Zirfas

\section{Therapeutik und Lebenskunst}

Eine psychologisch-philosophische Grundlegung

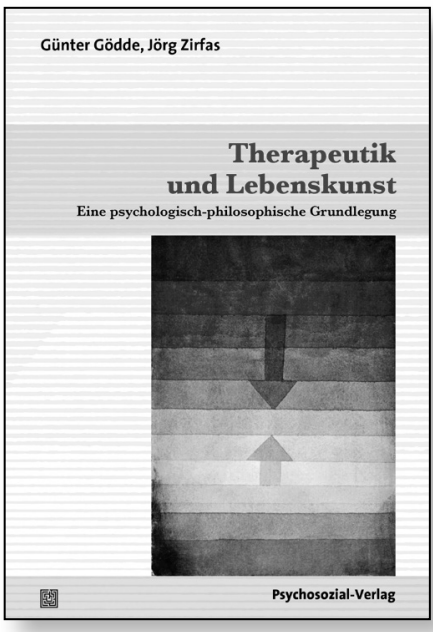

733 Seiten $\bullet$ Broschur $\bullet € 69,90$ ISBN 978-3-8379-2439-8

Therapeutik und Lebenskunst sind in vielfältiger Weise miteinander verknüpft. Lebenskunst ist in existenziellen Problemlagen auf therapeutische Erfahrungen und Praktiken angewiesen; umgekehrt sollte Psychotherapie das Deutungs-, Orientierungs- und Reflexionswissen der Lebenskunst heranziehen. Lebenskunst ohne Psychotherapie stellt eine praktische und Psychotherapie ohne Lebenskunst eine theoretische Verkürzung dar.

Günter Gödde und Jörg Zirfas beleuchten die zentralen Dimensionen der philosophischen und therapeutischen Lebenskunst und erörtern deren Bedeutung für die psychotherapeutische Praxis.

\section{Walltorstr. $10 \cdot 35390$ Gießen \\ Tel. 0641-969978-18 \\ Fax 0641-969978-19 \\ bestellung@psychosozial-verlag.de www.psychosozial-verlag.de}

